\title{
The growth inhibition of hepatocellular and cholangiocellular carcinoma cells by gemcitabine and the roles of extracellular signal-regulated and checkpoint kinases
}

\author{
KAZUYA MATSUMOTO, TAKAKAZU NAGAHARA, JUN-ICHI OKANO and YOSHIKAZU MURAWAKI
}

Second Department of Internal Medicine, Tottori University School of Medicine, 36-1 Nishi-cho, Yonago, Tottori 683-8504, Japan

Received March 13, 2008; Accepted June 25, 2008

DOI: $10.3892 /$ or_00000084

\begin{abstract}
We examined the effects of gemcitabine, a pyrimidine analogue, on hepatocellular carcinoma (HCC) and cholangiocellular carcinoma (CCC) cells. After HCC cells (HepG2, Hep3B, HLF and PLC/PRF/5) and CCC cells (HuCCT-1) were treated with gemcitabine, cellular growth, cell cycle, nuclear morphology and activity of signaling molecules were evaluated by WST- 8 assays, flow cytometry analysis, Hoechst 33258 staining and Western blotting, respectively. We found that gemcitabine significantly inhibited the growth of HCC and CCC cells in a dose- and time-dependent manner. Gemcitabine induced cell cycle arrest at the G1 phase, however, the sub-G1 fraction was not observed and nuclear morphology did not indicate the induction of apoptosis. Gemcitabine induced differential activation of checkpoint kinases, Chk2 and Chk1, in HCC and CCC cells, respectively and gemcitabine activated extracellular signal-regulated kinase (ERK) $1 / 2$ in both cell types. After the cells were pretreated with a MEK inhibitor U0126, activations of these checkpoint kinases were abrogated and the cell death was enhanced. These results demonstrate that gemcitabine inhibited the growth of HCC and CCC cells by cell cycle arrest without apoptosis and that the ERK/Chk1/2 signaling pathway was in part responsible for the resistance to gemcitabine. Our findings shed light on treating patients with $\mathrm{HCC}$ and CCC by gemcitabine, especially when combined with a MEK inhibitor and Chk1/2 inhibitors.
\end{abstract}

Correspondence to: Dr Jun-ichi Okano, Second Department of Internal Medicine, Tottori University School of Medicine, 36-1 Nishi-cho, Yonago, Tottori 683-8504, Japan

E-mail: okanoj@hotmail.co.jp

Abbreviations: CCC, cholangiocellular carcinoma; Chk, check point kinase; ERK, extracellular signal-regulated kinase; HCC, hepatocellular carcinoma; MAPK, mitogen-activated protein kinase; MEK, mitogen-activated ERK-regulating kinase

Key words: cholangiocellular carcinoma, hepatocellular carcinoma, gemcitabine, extracellular signal-regulated kinase, checkpoint kinases

\section{Introduction}

Hepatocellular carcinoma (HCC) is one of the most common malignancies in the world and the third-leading cause of death from cancer (1). The incidence of intrahepatic cholangiocellular carcinoma (CCC), the second most common tumor of primary liver cancers in adults, is also rising worldwide (2). Regardless of the advances in diagnostic and therapeutic procedures for these liver cancers, the prognosis of patients with HCC and CCC is still poor (3). A better understanding of the molecular mechanisms in the progression of HCC and $\mathrm{CCC}$ should contribute to the establishment of more effective therapies to these tumors.

Gemcitabine (2',2'-difluorocytidine monohydrochloride), a pyrimidine analogue, has been clinically utilized for the treatment of patients with pancreatic cancer (4) and nonsmall cell lung cancer (NSCLC) (5). In addition to these types of cancers, there is accumulating evidence that gemcitabine was effective in patients with advanced HCC (6-9) and intrahepatic CCC $(10,11)$, when used as a single agent and combined with other chemotherapeutic drugs such as platinum. These clinical data are encouraging for the clinical application of gemcitabine in the treatment of patients with advanced HCC and intrahepatic CCC.

The mechanisms of anti-proliferative action of gemcitabine have been mostly investigated in pancreatic cancer cells, including the induction of apoptosis mediated by the inhibition of pancreatitis-associated protein (PAP) and glycogen synthase kinase $3 ß$ (GSK-3ß) (12) and activation of p38 mitogen-activated protein kinase (MAPK) (13). In addition, nuclear factor $\kappa \mathrm{B}(\mathrm{NF}-\kappa \mathrm{B})(14)$ and bcl-2 (15) have been demonstrated to be relevant to the resistance to gemcitabine. However, the growth inhibitory effect and molecular mechanisms of gemcitabine on HCC and CCC cells are poorly understood. Our present data demonstrate that gemcitabine inhibited the growth of $\mathrm{HCC}$ and $\mathrm{CCC}$ cells by cell cycle arrest without apoptosis and that the extracellular signal-regulated kinase (ERK)/Chk1/2 signaling pathway was responsible for the resistance to gemcitabine, shedding light on treating patients with HCC and CCC by gemcitabine, particularly when combined with a MEK inhibitor and Chk1/2 inhibitors. 


\section{Materials and methods}

Materials. Gemcitabine was kindly provided by Eli Lilly Japan K.K. (Kobe, Japan) and dissolved in phosphate-buffered saline (PBS) as a vehicle. A phosphorylation-independent antibody for ERK1/2 was purchased from Stressgen Biotechnologies Corp. (Victoria, Canada). An antibody against the phosphorylated form of ERK1/2 at the Thr202/Tyr204 residues, an anti- $\beta$-actin antibody and Hoechst 33258 were purchased from Sigma (St. Louis, MO, USA). U0126, a MEK inhibitor, was purchased from Calbiochem-Novabiochem Corp. (San Diego, CA, USA), dissolved in DMSO as a vehicle and added to cells with a final concentration of $0.1 \%$ 30 min prior to the treatment with gemcitabine. The phosphorylation-specific Chk1/2 (Thr345 for Chk1 and Thr387 for Chk2, respectively), phosphorylation-independent Chk1/2 antibodies and phosphorylation-specific pRb antibody at the Ser795 residue were obtained from Cell Signaling Technology (Beverly, MA, USA). An anti-cyclin E antibody was obtained from Santa Cruz Biotechnology (Santa Cruz, CA, USA). Secondary anti-mouse and anti-rabbit horseradish peroxidase antibodies were obtained from GE Healthcare Ltd. (Buckinghamshire, UK).

Cell lines and tissue culture. All human liver cancer cells were purchased from American Type Culture Collection (Manassas, VA, USA). HCC cells (HepG2, Hep3B, HLF and $\mathrm{PLC} / \mathrm{PRF} / 5$ ) and intrahepatic CCC cells (HuCCT-1) were cultured in Dulbecco's modified Eagle's medium (DMEM) (Invitrogen Japan K.K., Tokyo, Japan) and RPMI-1640 medium (Cambrex Corp., East Rutherford, NJ, USA), respectively, supplemented with $10 \%$ fetal bovine serum (FBS) (Cosmo Bio Co., Ltd., Tokyo, Japan) and penicillin/ streptomycin (Cosmo Bio Co., Ltd.) at $37^{\circ} \mathrm{C}$ in a $5 \% \mathrm{CO}_{2}$ incubator.

Cell viability. Cell viability was assessed by WST- 8 assays (Cell counting Kit-8, Dojindo Corp., Kumamoto, Japan) following the supplier's protocol. Briefly, cells were grown in 96-well plates at $37^{\circ} \mathrm{C}$ in a $5 \% \mathrm{CO}_{2}$ incubator and treated with compounds. After $10 \mu \mathrm{l}$ of reagent was added, the optical density (OD) 450 was measured by a microplate reader (Dade Behring, Deerfield, IL, USA) and percentages of viable cells were compared to untreated control cells containing a vehicle. The experiments were repeated three times and data were expressed as the mean $\pm \mathrm{SD}$.

Hoechst 33258 staining. Cells grown in 8-well slides (Nalgen Nunc International, Rochester, NY, USA) were fixed in methanol/acetic acid (3:1) solution for $15 \mathrm{~min}$, washed with PBS for 5 min three times and stained with Hoechst 33258 solution (Sigma-Aldrich, St. Louis, MO, USA) for $20 \mathrm{~min}$ under light-protected condition. The cells were observed under fluorescence microscopy.

Flow cytometry. The cell pellets treated with gemcitabine were washed with PBS containing $1 \%$ FBS, fixed in $70 \%$ ethanol and stained with $50 \mu \mathrm{g} / \mathrm{ml}$ of propidium iodide (Sigma) containing 3 Kunitz RNase (Nippon Gene Co., Ltd, Toyama, Japan). Flow cytometry analysis was performed with a flow cytometer (Epics-XL, Beckman Coulter Inc., Miami, FL, USA).

Total protein preparation and Western blotting. Total protein preparation and Western blotting were conducted, as described previously (16). Briefly, cells were lysed in radioimmune precipitation (RIPA) buffer (Millipore Corp., MA, USA) supplemented with $1 \mathrm{mM}$ sodium orthovanadate, $1 \mathrm{mM}$ phenylmethylsulfonyl fluoride (PMSF) and a protease inhibitor mixture tablet (Roche Diagnostics, Basel, Switzerland) for $10 \mathrm{~min}$ on ice. Total protein samples $(10 \mu \mathrm{g})$ were separated on a sodium lauryl sulfate (SDS)-polyacrylamide gel (PAGE) and transferred to a polyvinylidene difluoride (PVDF) membrane (Immobilon-P, Millipore, Bedford, MA, USA). After the membranes were blocked in 5\% non-fat milk (Santa Cruz Biotechnology Inc.) in TBST (10 mM Tris, $150 \mathrm{mM} \mathrm{NaCl}, \mathrm{pH} 8.0$ and $0.1 \%$ Tween-20) for $1 \mathrm{~h}$ at room temperature, they were probed with primary antibodies overnight at $4^{\circ} \mathrm{C}$, washed three times in TBST, incubated with anti-mouse or anti-rabbit horseradish peroxidase (HRP) antibody in TBST for $1 \mathrm{~h}$ at room temperature. The signals were visualized using a chemiluminescence solution (ECL, GE Healthcare Ltd., Buckinghamshire, UK). The signals were quantified by a densitometry (Quantity One Basic, Bio-Rad Laboratories, Inc., Hercules, CA, USA).

Statistical analysis. Data were expressed as the mean values (columns) \pm SD (bars). The data of WST-8 assays were analyzed using the paired t-test to assess differences between experimental groups. Statistical significance was inferred at $\mathrm{P}<0.05$.

\section{Results}

Gemcitabine has an anti-proliferative effect on HCC and CCC cells. We first evaluated the effect of gemcitabine on the growth of primary human liver cancer cells including four HCC cell lines (HepG2, Hep3B, HLF and PLC/PRF/5) and one CCC cell line (HuCCT-1) by WST- 8 assays. We determined the concentrations of gemcitabine from $100 \mathrm{nM}$ to $1 \mathrm{mM}$, because the peak plasma concentration of gemcitabine when administered in patients with pancreatic cancer is $\sim 100 \mu \mathrm{M}$ (17). Increasing doses of gemcitabine inhibited the growth of both HCC and CCC cells in a dose- and timedependent manner (Fig. 1A-E). These results indicate that clinically relevant doses of gemcitabine inhibit the growth of primary liver cancer cells including HCC and CCC cells.

Effects of gemcitabine on cell cycle machinery and apoptosis. To gain insight into the mechanisms by which gemcitabine inhibited the growth of liver cancer cells, we examined the effect of gemcitabine on cell cycle machinery. Flow cytometry analysis revealed that cell cycle was arrested at the G0/G1 phase after Hep3B and HuCCT-1 cells were treated with gemcitabine and the subG1 fraction, a hallmark of apoptosis, was not induced after the gemcitabine treatment (Fig. 2A). Similar results were obtained in HepG2, HLF and PLC/PRF/5 (data not shown). When Hep3B cells were treated with $1 \mathrm{mM}$ gemcitabine, expression levels of cyclin E were significantly 
(A) HepG2
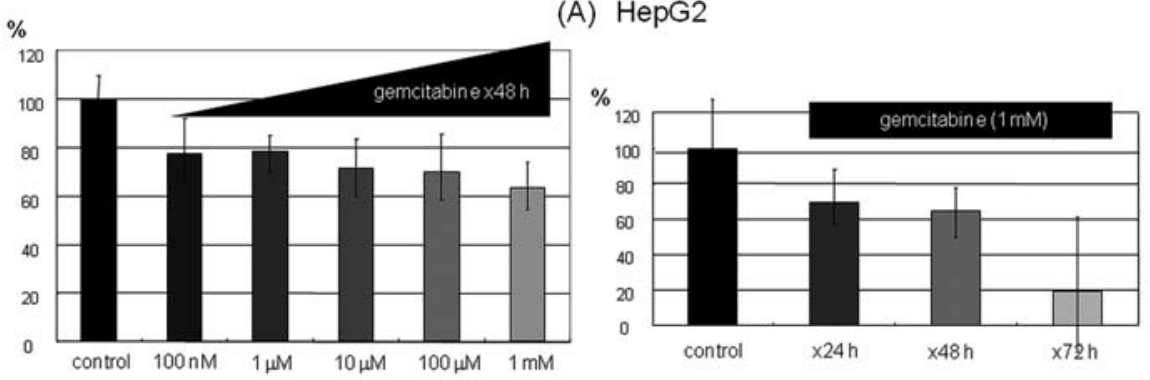

(B) Hep3B
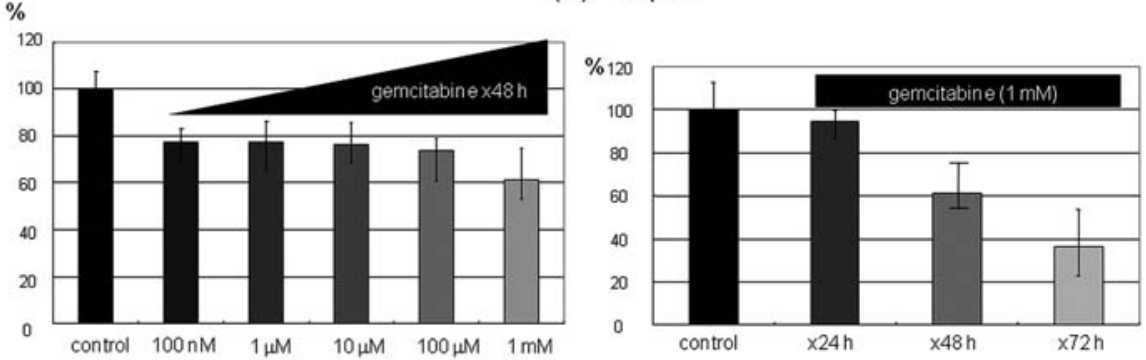

(C) HLF
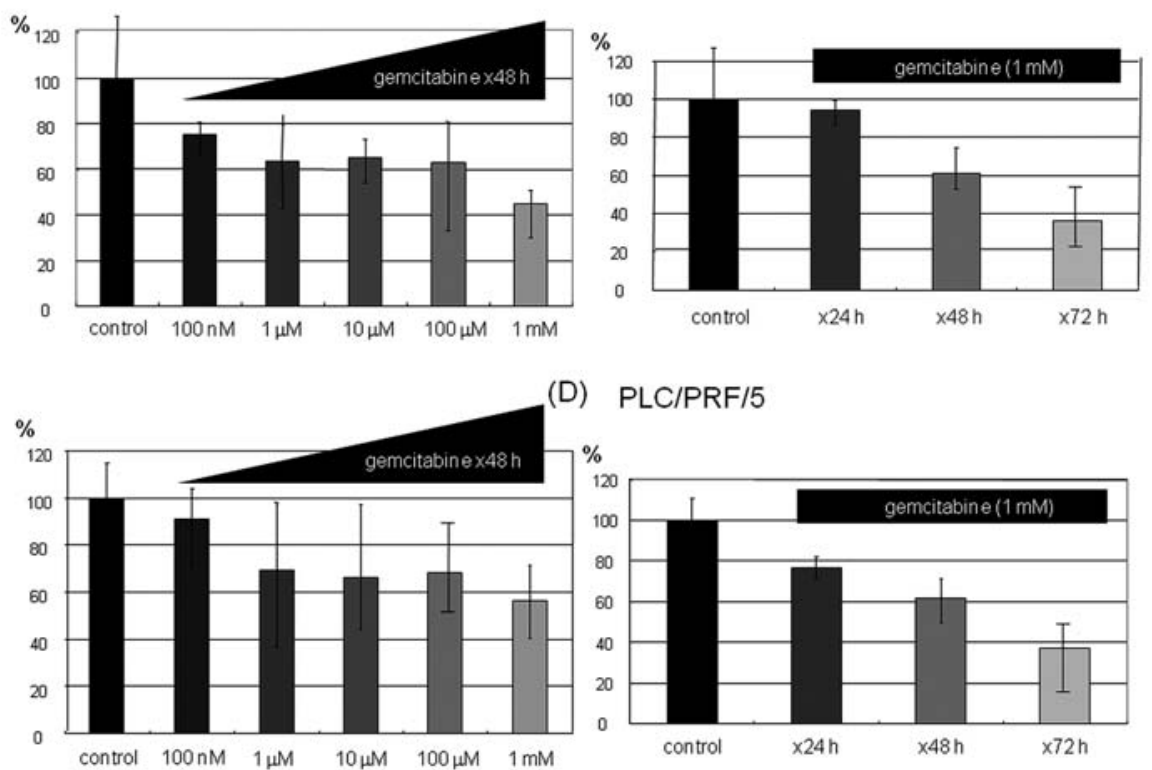

D) $\mathrm{PLC} / \mathrm{PRF} / 5$

(E) HuCCT-1
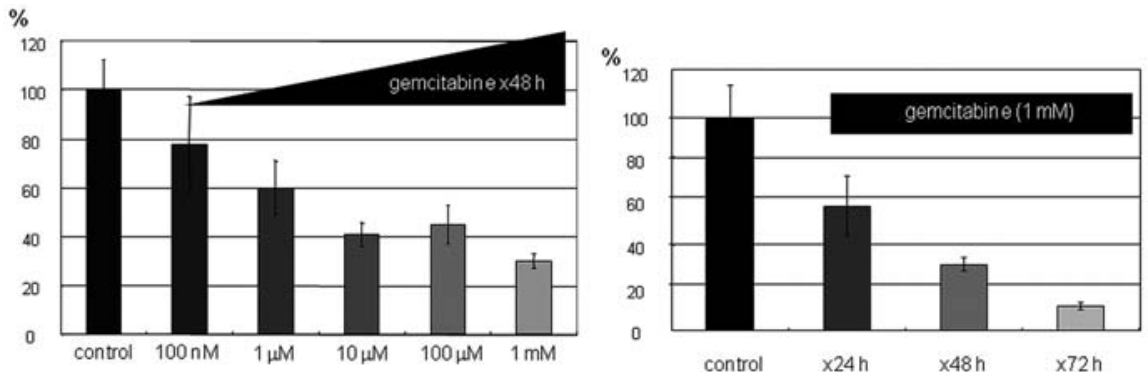

Figure 1. Growth inhibition of hepatocellular carcinoma (HCC) and cholangiocellular carcinoma (CCC) cells by gemcitabine. After hepatocellular carcinoma (HCC) cells (A) HepG2, (B) Hep3B, (C) HLF, (D) PLC/PRF/5 and CCC cells (E) HuCCT-1 were treated with increasing doses of gemcitabine (100 nM, $1 \mu \mathrm{M}$, $10 \mu \mathrm{M}, 100 \mu \mathrm{M}$ and $1 \mathrm{mM}$ ) for $48 \mathrm{~h}$, WST-8 assays were performed (left panels). These cells were treated with $1 \mathrm{mM}$ gemcitabine for 24,48 and $72 \mathrm{~h}$ and WST-8 assays were performed (right panels). The proliferation of cells treated with gemcitabine was compared to that of control cells treated with $0.1 \%$ phosphate-buffered saline (PBS). These experiments were repeated three times and data were expressed as the mean \pm SD. 
(A)
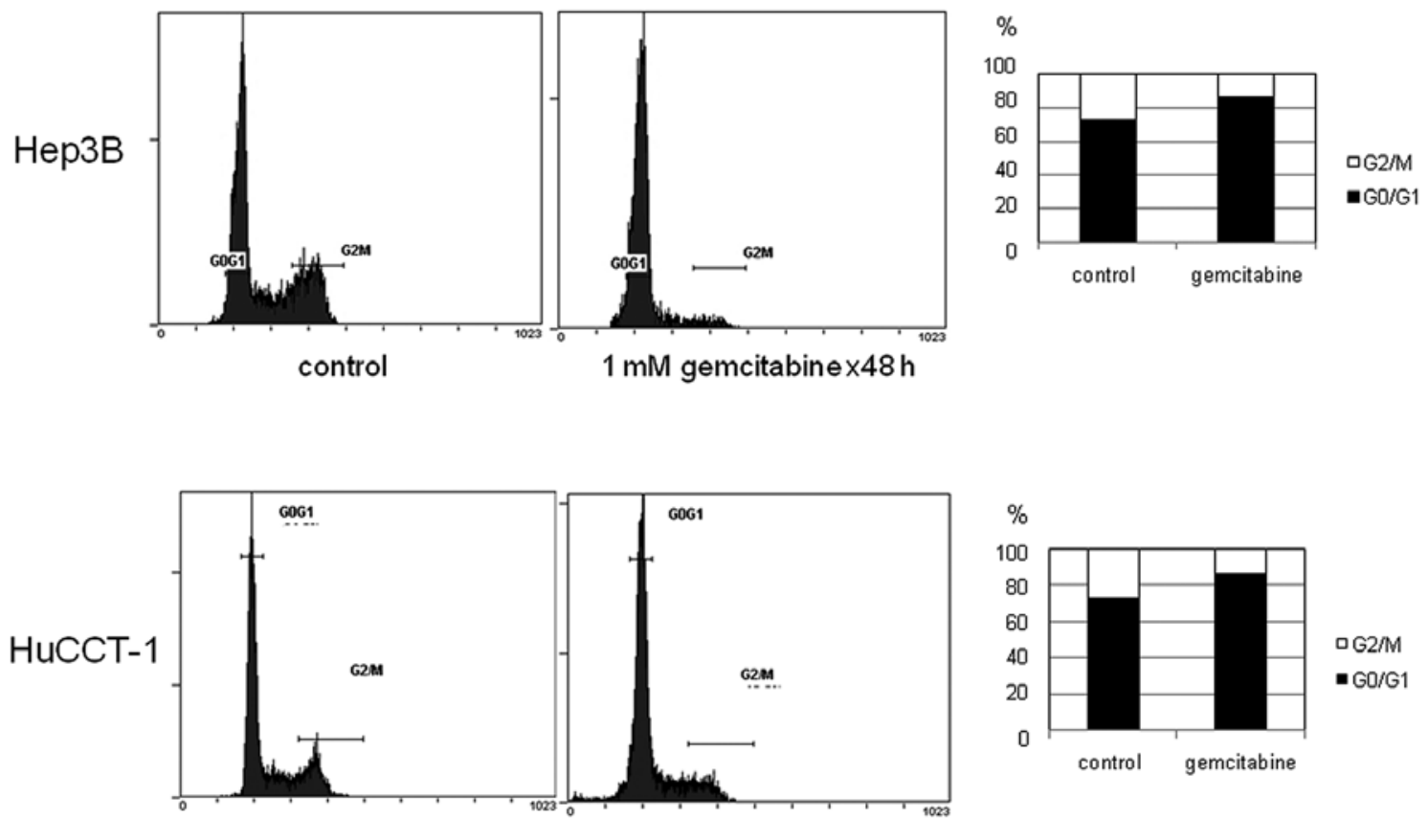

(B)

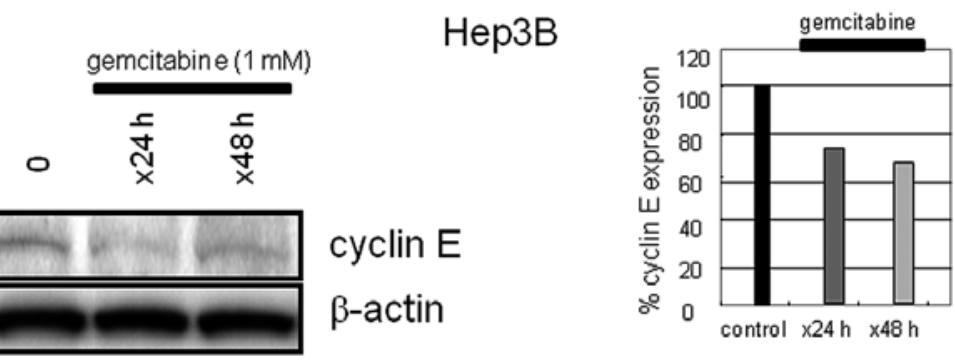

HLF

gemcitabine (1 mM)
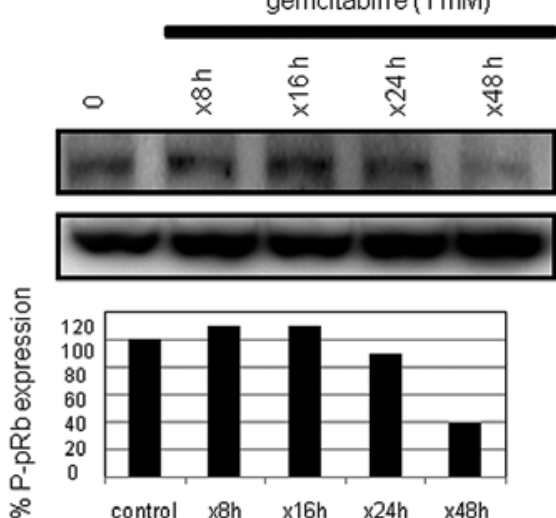

HuCCT-1

gemcitabine (1 mM)

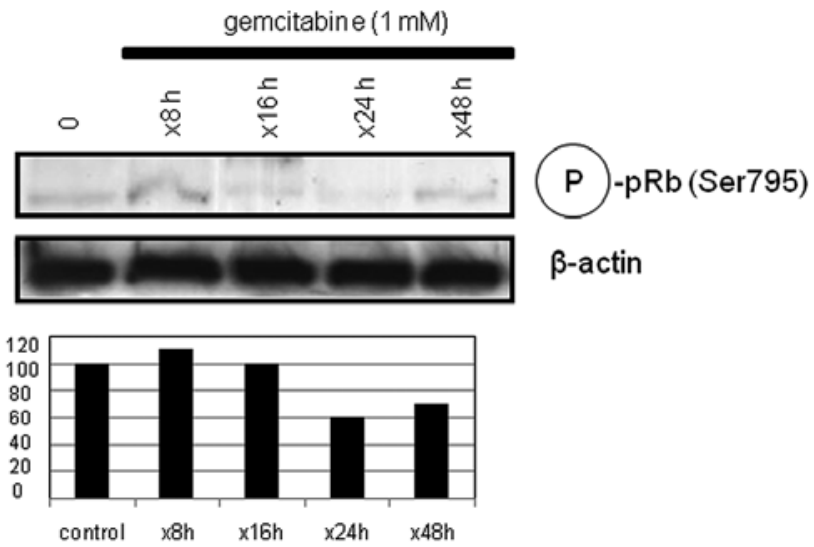

Figure 2. A and B. Effects of gemcitabine on cell cycle machinery and nuclear morphology. 
(C)

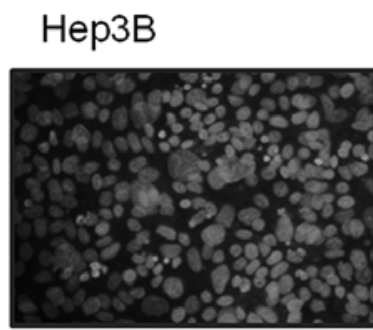

control

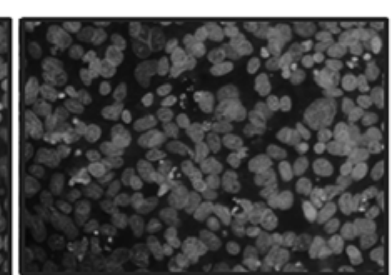

$\times 24 \mathrm{~h}$

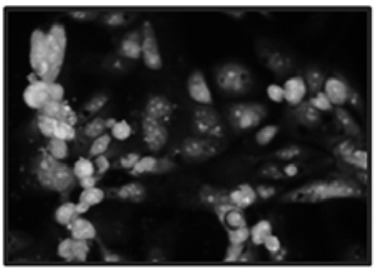

control

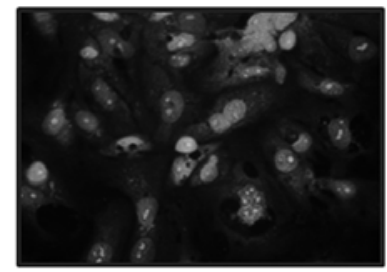

gemcitabine $(1 \mathrm{mM}) \times 48 \mathrm{~h}$

Figure 2. Effects of gemcitabine on cell cycle machinery and nuclear morphology. (A) HCC cells (Hep3B, top panel) and CCC cells (HuCCT-1, bottom panel) were treated with $1 \mathrm{mM}$ gemcitabine for $24 \mathrm{~h}$ and flow cytometry analysis was performed. Control cells were treated with $0.1 \%$ PBS. (B) (top panel) Western blot analysis was performed after Hep3B cells were treated with $1 \mathrm{mM}$ gemcitabine for 24 and $48 \mathrm{~h}$ and probed with the anti-cyclin E antibody. Control cells were treated with $0.1 \%$ PBS. Equal protein loading was confirmed by reprobing the membrane with the anti- $\beta$-actin antibody. (bottom panel) Western blot analysis was performed after HLF and HuCCT-1 cells were treated with $1 \mathrm{mM}$ gemcitabine for 8, 16, 24 and $48 \mathrm{~h}$, and probed with the phospho-pRb (Ser795) antibody. Control cells were treated with $0.1 \%$ PBS. Equal protein loading was confirmed by reprobing the membranes with the anti- $\beta$-actin antibody. The signals were quantified by a densitometry and normalized to the intensity of $\beta$-actin. (C) Nuclear morphology was examined by Hoechst 33258 staining after Hep3B (top panel) and HuCCT-1 cells (bottom panel) were treated with $1 \mathrm{mM}$ gemcitabine. Control cells contained $0.1 \%$ PBS as a solvent (original maginification x200).

decreased after 24 and $48 \mathrm{~h}$ of the treatment and phosphorylation of $\mathrm{pRb}$ was significantly inhibited after HLF cells and HuCCT-1 cells were treated with gemcitabine (Fig. 2B). Similar results were obtained in other HCC cells (data not shown). The present data suggested the involvement of cyclin $\mathrm{E}$ and $\mathrm{pRb}$ in cell cycle arrest by gemcitabine in these liver cancer cells.

We then examined nuclear morphology of liver cancer cells after challenged with gemcitabine. Gemcitabine treatment did not induce nuclear condensation and fragmentation, two hallmarks of apoptosis, in Hep3B and HuCCT-1 cells (Fig. 2C), suggesting that gemcitabine did not induce apoptosis.

Activation of ERK1/2 by gemcitabine treatment in liver cancer cells. We examined the effects of gemcitabine on signaling molecules in HCC and CCC cells. Since ERK1/2 has been associated with the regulation of cellular growth, apoptosis and chemoresistance $(18,19)$, we tested whether ERK1/2 activity was modulated by gemcitabine in the liver cancer cells. Western blot analysis revealed that ERK1/2 was significantly activated after Hep3B and HLF cells were treated with $1 \mathrm{mM}$ gemcitabine for 24 and $48 \mathrm{~h}$ (Fig. 3A). The ERK1/2 activation by gemcitabine was mediated by MEK based on the result that ERK1/2 activation was mostly inhibited by pretreating Hep3B cells with a MEK inhibitor U0126 (Fig. 3B). ERK1/2 was not activated in HepG2 and PLC/PRF/5 cells, suggesting that
ERK1/2 was activated in a cell-type specific manner. The ERK1/2 activation by gemcitabine was also observed in CCC cells HuCCT-1, although the time course of ERK1/2 activation was distinct from that of HCC cells, which peaked at 8 and $48 \mathrm{~h}$, and sustained until $72 \mathrm{~h}$ after the gemcitabine treatment (Fig. 3A).

We then investigated the roles of ERK $1 / 2$ activation induced by gemcitabine in the liver cancer cells. In WST-8 assays, a MEK inhibitor U0126 alone inhibited the growth of Hep3B and HuCCT-1 cells, revealing that MEK/ERK activity was necessary for the basal growth of these cells (Fig. 3C). When the cells were pretreated with $50 \mu \mathrm{M}$ U0126 followed by treatment with $1 \mathrm{mM}$ gemcitabine for $48 \mathrm{~h}$, the cell death induced by gemcitabine was significantly accelerated in both Hep3B and HuCCT-1 cells (Fig. 3C). Similar results were obtained in HLF cells (data not shown). These data suggest that ERK1/2 may be a signaling molecule relevant to the resistance of HCC and CCC cells to the cell death by gemcitabine.

Differential regulation of Chk1 and Chk2 by gemcitabine in HCC and CCC cells. Chk1 and Chk2 are serine/threonine kinases activated (phosphorylated) in response to DNA damage in cell cycle events for protecting cells (20). Since the regulation of these checkpoint kinases in response to gemcitabine has not been fully investigated yet, we examined whether Chk1 and Chk2 are activated by gemcitabine in HCC 
(A)

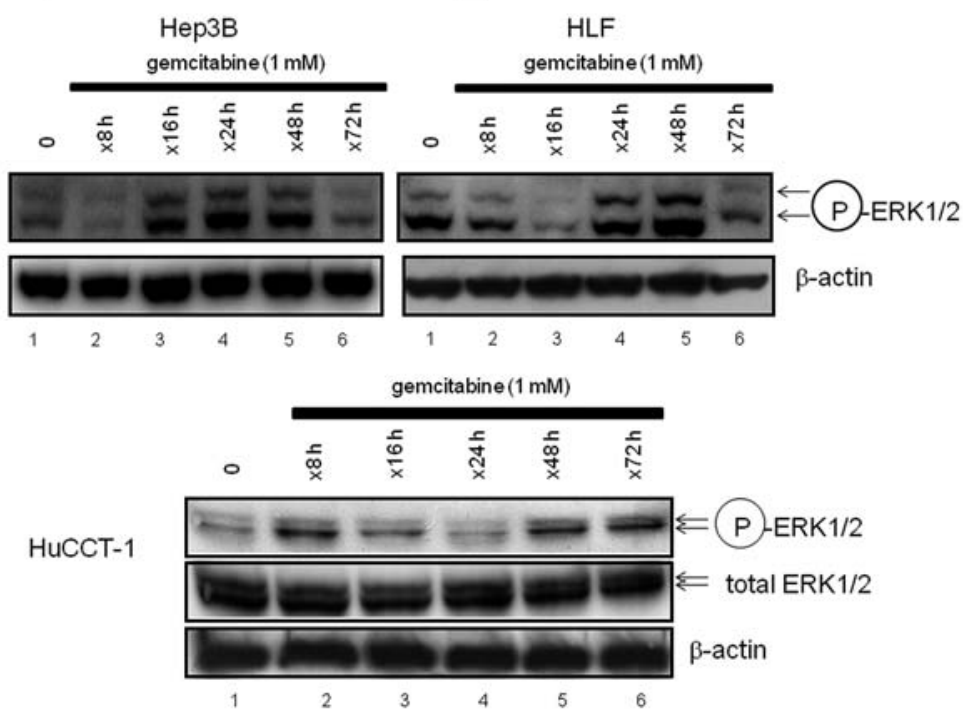

(B)

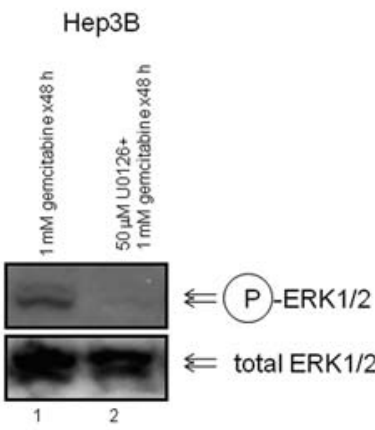

(C)
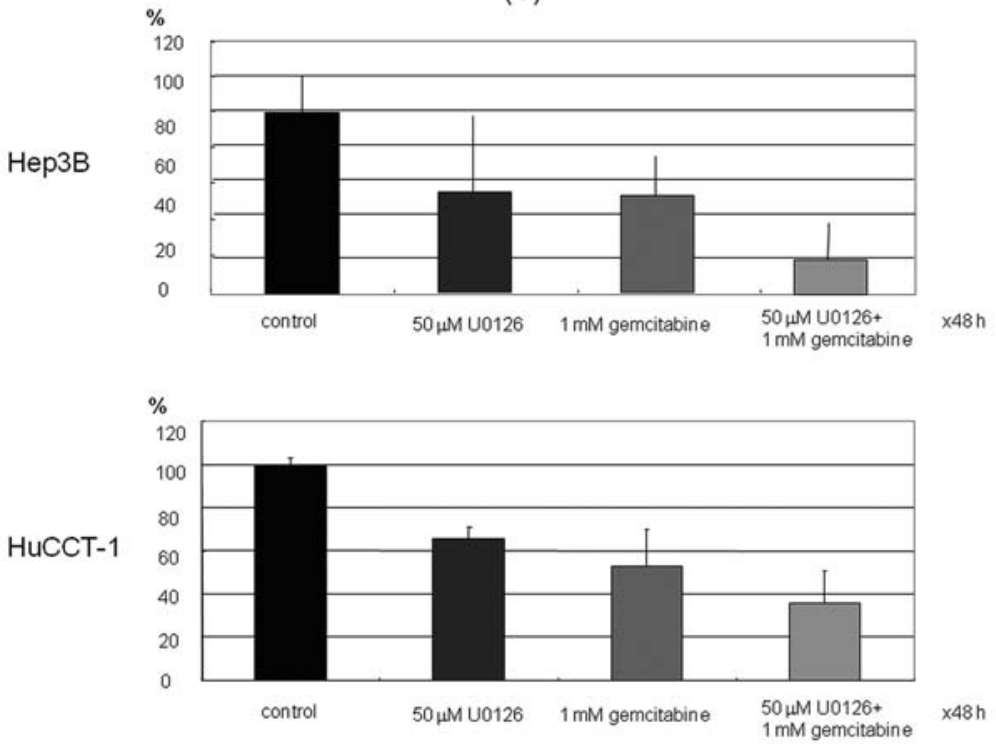

Figure 3. Modulation of extracellular signal-regulated kinase (ERK)1/2 activity by gemcitabine in HCC and CCC cells. (A) HCC cells (Hep3B and HLF) (top panel) and CCC cells (HuCCT-1) (bottom panel) were treated with $1 \mathrm{mM}$ gemcitabine for 8, 16, 24, 48 and $72 \mathrm{~h}$ and Western blot analysis was performed with the phospho-extracellular signal-regulated kinase (ERK)1/2 (Thr202/Tyr204) antibody. The upper and lower bands are ERK1 and ERK2, respectively (arrows). The membranes were reprobed with the anti-B-actin antibody (bottom panel). (B) After Hep3B cells were treated with $1 \mathrm{mM}$ gemcitabine and pretreated with $50 \mu \mathrm{M}$ U0126 followed by $1 \mathrm{mM}$ gemcitabine for $48 \mathrm{~h}$, Western blot analysis was performed with the phospho-ERK1/2 (Thr202/Tyr204) antibody (top panel). The membrane was reprobed with the total ERK1/2 antibody (bottom panel). The upper and lower bands are ERK1 and ERK2, respectively (arrows). (C) WST-8 assays were performed after Hep3B cells (top panel) and HuCCT-1 cells (bottom panel) were treated with 50 $\mu \mathrm{M}$ U0126, $1 \mathrm{mM}$ gemcitabine and pretreated with $50 \mu \mathrm{M}$ U0126 followed by $1 \mathrm{mM}$ gemcitabine for $48 \mathrm{~h}$. Control cells were treated with $0.1 \%$ DMSO and PBS. These experiments were repeated three times and data are expressed as the mean $\pm \mathrm{SD}$. 
(A)

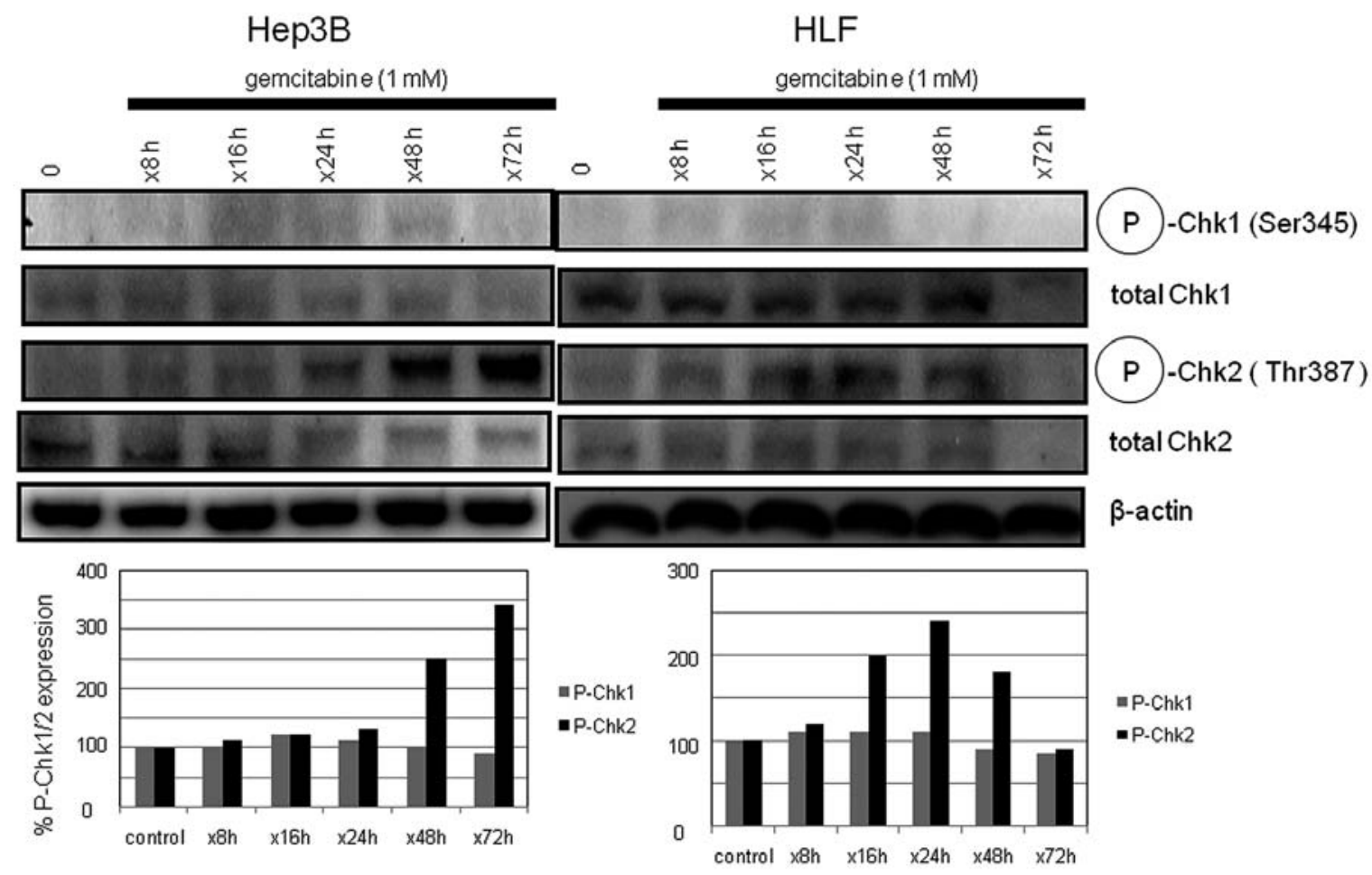

(B)

HuCCT-1

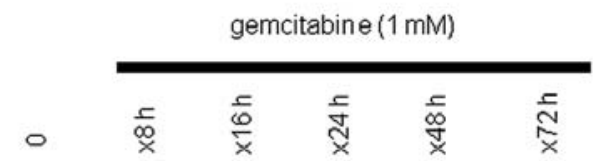

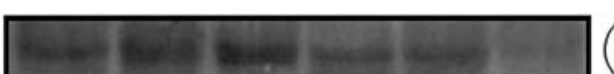

(P)-Chk1 (Ser345)

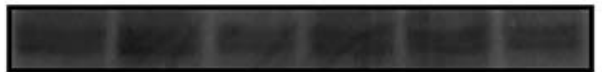

total Chk1

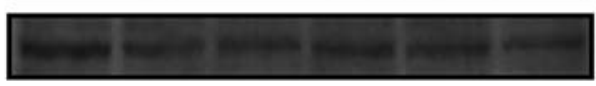

(P)-Chk2 (Thr387)

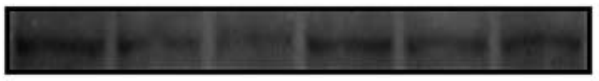

total Chk2

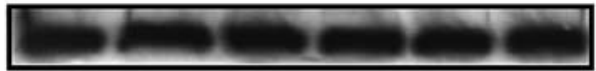

$\beta$-actin

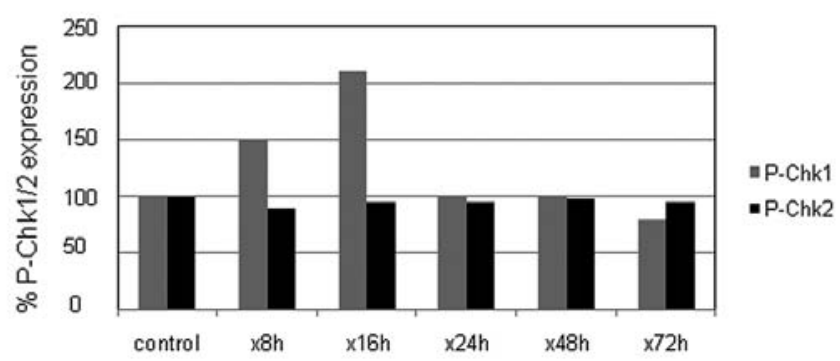

Figure 4. A and B. Differential activation of checkpoint kinases by gemcitabine in HCC and CCC cells. 
(C)

\section{Hep3B}

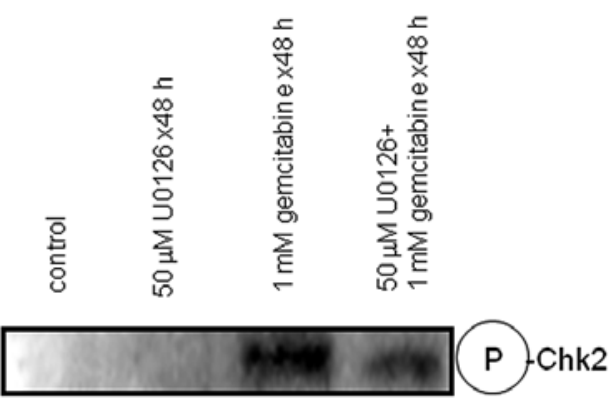

total Chk2

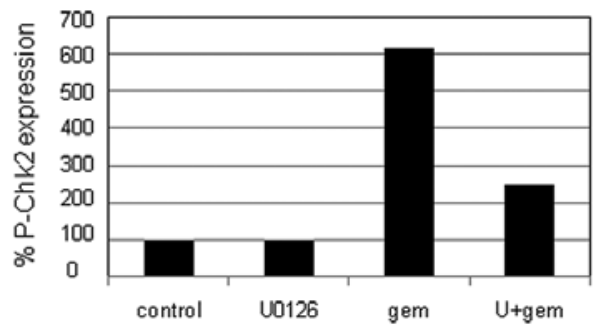

HuCCT-1
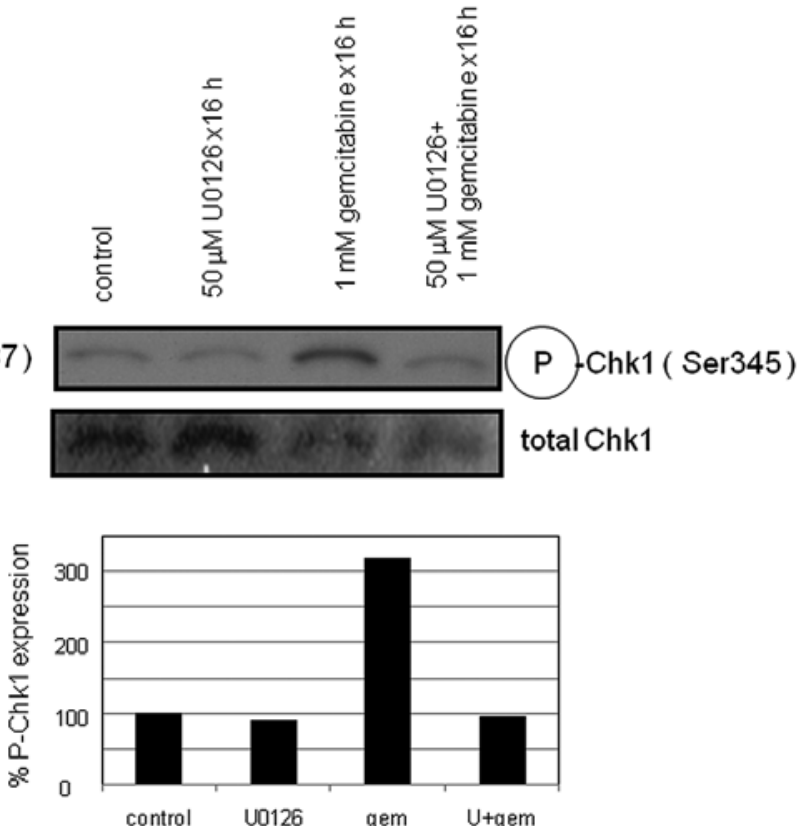

Figure 4. Differential activation of checkpoint kinases by gemcitabine in HCC and CCC cells. (A) HCC cells (Hep3B and HLF) were treated with 1 mM gemcitabine for 8, 16, 24, 48 and $72 \mathrm{~h}$ and Western blot analysis was performed with the phospho-Chk1 (Ser345) (top panel), total Chk1 (2nd panel), phospho-Chk2 (Thr387) and total Chk2 (4th panel) antibodies. The membranes were reprobed with the anti- $\beta$-actin antibody (bottom panel). The signals were quantified by a densitometry and normalized to the intensity of $\beta$-actin. (B) CCC cells (HuCCT-1) were treated with 1 mM gemcitabine for $8,16,24,48$ and $72 \mathrm{~h}$ and Western blot analysis was performed with the phospho-Chk1 (Ser345) (top panel), total Chk1 (2nd panel), phospho-Chk2 (Thr387) and total Chk2 (4th panel) antibodies. The membranes were reprobed with the anti-ß-actin antibody (bottom panel). The signals were quantified by a densitometry and normalized to the intensity of B-actin. (C) (left panel) Hep3B cells were treated with $50 \mu \mathrm{M}$ U0126, 1 mM gemcitabine and pretreated with $50 \mu \mathrm{M}$ U0126 followed by $1 \mathrm{mM}$ gemcitabine for $48 \mathrm{~h}$, control cells were treated with $0.1 \%$ DMSO and PBS and Western blot analysis was conducted with the phospho-Chk2 (Thr387) antibody (top panel). The membrane was reprobed with the total Chk2 antibody (bottom panel). (right panel) HuCCT-1 cells were treated with $50 \mu \mathrm{M}$ U0126, $1 \mathrm{mM}$ gemcitabine and pretreated with $50 \mu \mathrm{M}$ U0126 followed by $1 \mathrm{mM}$ gemcitabine for $16 \mathrm{~h}$, control cells were treated with $0.1 \%$ DMSO and PBS and Western blot analysis was conducted with the phospho-Chk1 (Ser345) antibody (top panel). The membrane was reprobed with the total Chk1 antibody (bottom panel). The signals were quantified by a densitometry and normalized to the intensity of total Chk1/2.

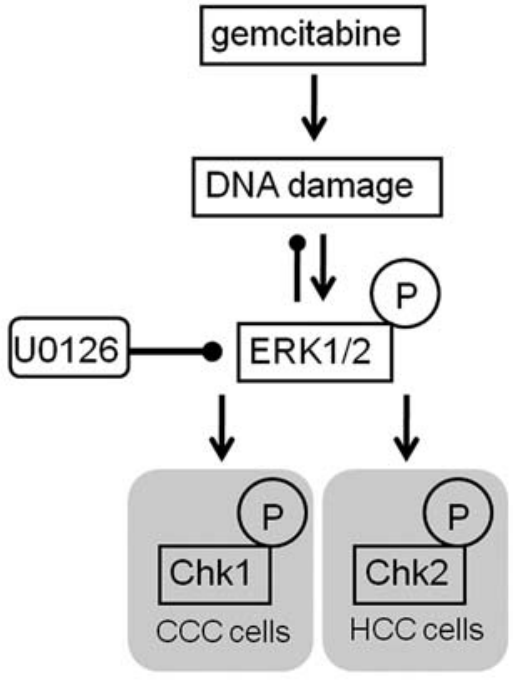

Figure 5. Schematic presentation of signaling molecules modulated by gemcitabine in HCC and CCC cells. Signaling pathways examined in the present study are summarized. Gemcitabine induced cell death by DNA damage, which activated ERK1/2 via MEK in HCC and CCC cells. In HCC and CCC cells, Chk2 and Chk1 were activated in response to gemcitabine treatment, respectively, which was mediated by ERK1/2 activation. and $\mathrm{CCC}$ cells. The phosphorylation of $\mathrm{Chk} 2$ was induced in two HCC cells Hep3B and HLF after 48 and $16 \mathrm{~h}$ of the gemcitabine treatment, respectively, although the phosphorylation of Chk1 was marginally observed in either cells (Fig. 4A). Interestingly, in contrast to HCC cells, Chk1 was significantly phosphorylated in HuCCT-1 and CCC cells, in response to the gemcitabine treatment, although phosphorylation levels of Chk2 were not significantly modulated (Fig. 4B).

We finally examined the involvement of ERK1/2 activity in the activation of these checkpoint kinases. When Hep3B and HuCCT-1 cells were pretreated with a MEK inhibitor U0126, phosphorylation levels of Chk2 and Chk1 induced by gemcitabine were significantly inhibited in each cell line, respectively (Fig. 4C). These results indicate that ERK $1 / 2$ is an upstream molecule responsible for the Chk1/2 activations, and that this signaling pathway may play a role in resisting the DNA damage caused by gemcitabine (Fig. 5).

\section{Discussion}

Gemcitabine is a pyrimidine analogue clinically utilized for the treatment of patients with pancreatic cancer (4) and 
NSCLC (5). HCC and CCC are primary liver cancer with dismal prognosis. Based on accumulating evidence that gemcitabine was beneficial to patients with advanced HCC (6-9) and intrahepatic CCC $(10,11)$, we sought to examine the biological effects of gemcitabine on HCC and CCC cells in vitro.

The doses used in this study are clinically relevant, since the peak plasma concentration of gemcitabine in patients with pancreatic cancer is $\sim 100 \mu \mathrm{M}$ (17). When HCC and $\mathrm{CCC}$ cells were treated with these clinically relevant doses, anti-proliferative action was observed in vitro in concert with the recent clinical case reports (6-11). Our present study demonstrated that gemcitabine inhibited the proliferation of liver cancer cells via cell cycle arrest associated with decreased expression of cyclin $\mathrm{E}$ and phosphorylation of $\mathrm{pRb}$ and not by apoptosis. Although our result is contrary to previous findings in pancreatic cancer cells demonstrating an induction of apoptosis by gemcitabine $(12,13)$, the mechanisms by which gemcitabine exert an anti-proliferative action could be distinct depending on the cell types.

MAPKs have been linked to chemoresistance in pancreatic cancer cells (21) and activation of ERK1/2 by gemcitabine, which was a promoting factor of apoptosis, has been reported in NSCLC (22). We have demonstrated that gemcitabine activated ERK1/2 in HCC and CCC cells, which resisted the cell death by gemcitabine, because abrogation of ERK $1 / 2$ by a MEK inhibitor U0126 accelerated the cells death. These discrepant roles of activated ERK1/2 between the cell lines are not surprising, because ERK1/2 activation may exert either proliferative or anti-proliferative influence depending on the cellular context (23). The time courses of ERK1/2 activation were fluctuated and distinct depending on the cell types. We speculate that ERK1/2 phosphatases regulating the ERK1/2 phosphorylation may be distinct between early and late time points and among cell lines as well.

Activation of cell-cycle checkpoints in response to various types of DNA damage is essential for the maintenance of genomic stability in eukaryotic cells (24). Checkpoint kinases Chk1 and Chk2 are structurally unrelated yet functionally overlapping serine/threonine kinases activated in response to DNA damage in cell cycle events for protecting cells (20). The phosphatidylinositol 3-kinase (PI3K) family ataxia telangiectasia and Rad3 related (ATR) and ataxia telangiectasia mutated (ATM) are upstream kinases of Chk1 and Chk2, respectively (25). Activation of these checkpoint kinases in response to gemcitabine and roles of activated Chk1 and Chk2 in cell cycle arrest have recently been demonstrated $(26,27)$. Notably, we observed differential activation of Chk1 and Chk2 in response to gemcitabine in CCC and HCC cells. Because Chk1 and Chk2 have redundant roles in part (28) and their regulatory mechanisms have not been investigated yet, the precise roles and regulation of differentially activated these checkpoint kinases need to be clarified in future studies.

Although crosstalk between the checkpoint kinases and MAPKs has been poorly investigated, involvement of p38MAPK on activation of Chk 1 and Chk2 has been demonstrated (24). In the present study, we have made a novel finding that ERK1/2 is an upstream molecule responsible for activation of the checkpoint kinases. Based on our data, combinatory treatment with gemcitabine and inhibitors to
MEK/ERK and checkpoint kinases could provide a new tool for treating patients with HCC and CCC, although the clinical relevance of these findings needs to be confirmed.

\section{Acknowledgements}

We would like to thank Eli Lilly Japan K.K. (Kobe, Japan) for kindly donating gemcitabine and Mr. Yujirou Ikuta and Mrs. Yumiko Koda for their technical assistance and support in conducting experiments.

\section{References}

1. Bosch FX, Ribes J and Borras J: Epidemiology of primary liver cancer. Semin Liver Dis 19: 271-285, 1999.

2. West J, Wood H, Logan RF, Quinn M and Aithal GP: Trends in the incidence of primary liver and biliary tract cancers in England and Wales 1971-2001. Br J Cancer 94: 1751-1758, 2006.

3. Huether A, Hopfner M, Baradari V, Schuppan D and Scherubl H: Sorafenib alone or as combination therapy for growth control of cholangiocarcinoma. Biochem Pharmacol 73: 1308-1317, 2007.

4. Burris HA III, Moore MJ, Andersen J, et al: Improvements in survival and clinical benefit with gemcitabine as first-line therapy for patients with advanced pancreas cancer: a randomized trial. J Clin Oncol 15: 2403-2413, 1997.

5. Martoni A, Marino A, Sperandi F, et al: Multicentre randomized phase III study comparing the same dose and schedule of cisplatin plus the same schedule of vinorelbine or gemcitabine in advanced non-small cell lung cancer. Eur J Cancer 41: 81-92, 2005.

6. Louafi S, Boige V, Ducreux M, et al: Gemcitabine plus oxaliplatin (GEMOX) in patients with advanced hepatocellular carcinoma (HCC): results of a phase II study. Cancer 109: 1384-1390, 2007.

7. Pastorelli D, Cartei G, Zustovich F, et al: Gemcitabine and liposomal doxorubicin in biliary and hepatic carcinoma (HCC) chemotherapy: preliminary results and review of the literature. Ann Oncol 17: v153-v157, 2006.

8. Parikh PM, Fuloria J, Babu G, et al: A phase II study of gemcitabine and cisplatin in patients with advanced hepatocellular carcinoma. Trop Gastroenterol 26: 115-118, 2005.

9. Ulrich-Pur H, Kornek GV, Fiebiger W, Schull B, Raderer M and Scheithauer W: Treatment of advanced hepatocellular carcinoma with biweekly high-dose gemcitabine. Oncology 60: 313-315, 2001

10. Kirchhoff T, Zender L, Merkesdal S, et al: Initial experience from a combination of systemic and regional chemotherapy in the treatment of patients with nonresectable cholangiocellular carcinoma in the liver. World J Gastroenterol 11: 1091-1095, 2005.

11. Vogl TJ, Schwarz W, Eichler K, et al: Hepatic intraarterial chemotherapy with gemcitabine in patients with unresectable cholangiocarcinomas and liver metastases of pancreatic cancer: a clinical study on maximum tolerable dose and treatment efficacy. J Cancer Res Clin Oncol 132: 745-755, 2006.

12. Jiang PH, Motoo Y, Sawabu N and Minamoto T: Effect of gemcitabine on the expression of apoptosis-related genes in human pancreatic cancer cells. World J Gastroenterol 12: 1597-1602, 2006.

13. Koizumi K, Tanno S, Nakano Y, et al: Activation of p38 mitogen-activated protein kinase is necessary for gemcitabineinduced cytotoxicity in human pancreatic cancer cells. Anticancer Res 25: 3347-3353, 2005.

14. Arlt A, Gehrz A, Muerkoster S, et al: Role of NF-kappaB and $\mathrm{Akt} / \mathrm{PI} 3 \mathrm{~K}$ in the resistance of pancreatic carcinoma cell lines against gemcitabine-induced cell death. Oncogene 22: 3243-3251, 2003.

15. Okamoto K, Ocker M, Neureiter D, et al: bcl-2-specific siRNAs restore Gemcitabine sensitivity in human pancreatic cancer cells. J Cell Mol Med 11: 349-361, 2007.

16. Okano J, Nagahara T, Matsumoto K and Murawaki Y: The growth inhibition of liver cancer cells by paclitaxel and the involvement of extracellular signal-regulated kinase and apoptosis. Oncol Rep 17: 1195-1200, 2007. 
17. Cattel L, Airoldi M, Passera R, Cagliero E, Stella B and Goffredo F: Gemcitabine plus vinorelbine chemotherapy regimens: a pharmacokinetic study of alternate administration sequences. Pharm World Sci 26: 238-241, 2004.

18. MacKeigan JP, Collins TS and Ting JP: MEK inhibition enhances paclitaxel-induced tumor apoptosis. J Biol Chem 275: 38953-38956, 2000.

19. Wada T and Penninger JM: Mitogen-activated protein kinases in apoptosis regulation. Oncogene 23: 2838-2849, 2004.

20. Bartek J and Lukas J: Chk1 and Chk2 kinases in checkpoint control and cancer. Cancer Cell 3: 421-429, 2003.

21. Zhao Y, Shen S, Guo J, et al: Mitogen-activated protein kinases and chemoresistance in pancreatic cancer cells. J Surg Res 136: 325-335, 2006.

22. Chang GC, Hsu SL, Tsai JR, Wu WJ, Chen CY and Sheu GT: Extracellular signal-regulated kinase activation and Bcl-2 downregulation mediate apoptosis after gemcitabine treatment partly via a p53-independent pathway. Eur J Pharmacol 502: $169-183,2004$

23. Okano J and Rustgi AK: Paclitaxel induces prolonged activation of the Ras/MEK/ERK pathway independently of activating the programmed cell death machinery. J Biol Chem 276: 19555-19564, 2001.
24. Reinhardt HC, Aslanian AS, Lees JA and Yaffe MB: p53deficient cells rely on ATM- and ATR-mediated checkpoint signaling through the $\mathrm{p} 38 \mathrm{MAPK} / \mathrm{MK} 2$ pathway for survival after DNA damage. Cancer Cell 11: 175-189, 2007.

25. Roos WP and Kaina B: DNA damage-induced cell death by apoptosis. Trends Mol Med 12: 440-450, 2006.

26. Morgan MA, Parsels LA, Parsels JD, Mesiwala AK, Maybaum J and Lawrence TS: Role of checkpoint kinase 1 in preventing premature mitosis in response to gemcitabine. Cancer Res 65: 6835-6842, 2005.

27. Karnitz LM, Flatten KS, Wagner JM, et al: Gemcitabine-induced activation of checkpoint signaling pathways that affect tumor cell survival. Mol Pharmacol 68: 1636-1644, 2005.

28. Bartek J and Lukas J: Mammalian G1- and S-phase checkpoints in response to DNA damage. Curr Opin Cell Biol 13: 738-747, 2001 